\title{
Age-related decline in item but not spatiotemporal associative memory for a real-world event
}

Nicholas B. Diamond ${ }^{1,2}$, Kristoffer Romero ${ }^{1}$, Nivethika Jeyakumar ${ }^{1}$, Brian Levine ${ }^{1,2,3}$

${ }^{1}$ Rotman Research Institute, Baycrest Health Sciences

${ }^{2}$ Department of Psychology, University of Toronto

${ }^{3}$ Department of Medicine (Neurology), University of Toronto 


\section{Abstract}

Normal aging is typically associated with reduced ability to reconstruct the spatiotemporal context of past events, a core component of episodic memory. However, little is known about our ability to remember the order of events comprising extended real-world experiences and how this ability changes with age. We leveraged the richness and structure of a museum exhibit to address this question. Three months after visiting the exhibit, 141 adults aged 18-84 completed a test of spatiotemporal order memory and old/new recognition using pictures from the exhibit and similar lures, from which measures of associative and item memory were derived. Order discrimination accuracy was modulated by inter-item order and distance in younger and older adults, extending findings from recognition of laboratory stimuli at short delays to remote realworld experiences. In contrast to established findings from laboratory-based assessments, we observed a significant effect of aging on item memory driven by increased lure susceptibility, but no age-related reduction in spatiotemporal associative memory. These findings present novel insights into different components of memory for real-world experiences at naturalistic timescales and across the lifespan. 


\section{Introduction}

Episodic memory is defined by the ability to recover the spatial and temporal context of specific past experiences (Tulving, 1972). Age-related reductions in memory for spatiotemporal contextual details are observed for both laboratory (e.g. Fabiani \& Friedman, 1997; Allen, Morris, Stark, Fortin, \& Stark, 2015) and naturalistic events (e.g., Levine et al., 2002), yet there is little connection between the methods used across these two testing platforms. Laboratory methods can objectively measure and manipulate the accuracy and spatiotemporal organization of retrieval with respect to the encoded episodes, but sequences of laboratory stimuli bear little resemblance to the sequences of events, places and actions that define everyday experiences. On the other hand, studies of real-life events (i.e., autobiographical memory) typically rely on free recall or subjective ratings that preclude objective measurement. Heterogeneity in the remoteness, content and context of participants' personal autobiographical memories exacerbates this problem, especially in the context of cognitive aging studies. Consequently, still little is known about how we retrace trajectories through past realworld experiences and how this ability changes with age.

Naturalistic events unfold over time in rich and immersive spatial contexts. Memory for such events can reveal crucial component processes of episodic memory, particularly the reconstruction of dynamic spatiotemporal representations (Buzsáki \& Llinás, 2017). Across species, spatial and temporal contexts form scaffolds for episodic memories (Ranganath \& Hsieh, 2016; Robin, Wynn, \& Moscovitch, 2015) and they are shaped by actions, goals and exploration (Shapiro, Kennedy, \& Ferbinteanu, 2006; 
Eichenbaum \& Cohen, 2014). In particular, properties characteristic of naturalistic encoding such as volitional control, self-initiated movement, curiosity and goaldirectedness modulate hippocampal-neocortical interactions that support episodic memory encoding, and increase subsequent retrieval performance relative to passive encoding baselines (Cornwell, Johnson, Holroyd, Carver, \& Grillon, 2008; Gruber, Gelman \& Ranganath, 2014; Kaplan et al., 2012; Voss, Gonsalves, Federmeier, Tranel, \& Cohen, 2011). These and other related processes are often studied in isolation in the laboratory but are jointly constitutive of everyday experiences. Furthermore, direct experience in real-world space produces qualitatively different spatial memory representations than desktop paradigms (virtual reality and video; Hegarty et al., 2006). Consequently, it is not surprising that autobiographical and laboratory memory tasks sometimes show little overlap in terms of brain activity patterns (McDermott et al., 2017), with greater activity in regions related to spatial reconstruction during retrieval of autobiographical episodes than even detail-rich videos (St-Laurent, Moscovitch, \& McAndrews, 2016), and hippocampal recordings in rodents and primates reveal different firing patterns during real-world versus virtual exploration (Aghajan et al., 2015; Thome et al., 2017). Thus, certain mechanisms supporting spatiotemporal context encoding and retrieval may be systematically missed by conventional laboratory encoding paradigms.

To bridge the gap between laboratory and naturalistic methods for investigating spatiotemporal information in episodic memory, researchers have recently used verifiable real-world encoding paradigms (Griffiths, Mazaheri, Debener, \& Hanslmayr, 
2016; Nielson et al., 2015; St. Jacques, Rubin, LaBar, \& Cabeza, 2008), although aging effects in such methods remain largely unexplored. In the present study, we used an exhibit at the Ontario Science Centre ("Brain: The Inside Story") as a rich, dynamic, large-scale and yet controlled one-shot episode for investigating spatiotemporal context memory in a sample of healthy adults ranging in age from 18-84. As a unique and precisely dated event, we were able to probe memory at $2-4$ months post-encoding, a critical period for memory consolidation (when extrapolated from animal research; Bontempi, Laurent-Demir, Destrade, \& Jaffard, 1999; Winocur, Moscovitch, Caruana, \& Binns, 2005; see Sheldon \& Levine, 2013) that is rarely tested in most laboratory methods (where memory is usually assessed at seconds- or minutes-long delays) or in autobiographical memory paradigms, where highly personally significant events are often probed years or decades after their occurrence.

As an ancillary goal, we also investigated whether order memory accuracy was sensitive to the spatiotemporal distance between items encountered in the exhibit, as has been previously shown at both laboratory (stimuli separated by seconds) and autobiographical (events separated by days-to-years) timescales (e.g. Curran \& Friedman, 2003; Skowronski, Walker, \& Betz, 2003; St. Jacques, Rubin, LaBar, \& Cabeza, 2008; Underwood, 1977), and whether distance sensitivity changed with age (Fabiani \& Friedman, 1997; Perlmutter, Metzger, Nezworksi, \& Miller, 1981; Roberts et al., 2014; Tolentino et al., 2012). An effect of distance on ordinal discrimination of the exhibit items at our months-long delay would provide novel evidence regarding the durability of memory for spatiotemporal relations within single extended events. 
Using photographs from the exhibit along with lures from a separate but similar exhibit, we devised a novel online test of old/new recognition and spatiotemporal order memory, allowing us to test predictions concerning age-related deficits for associative (context) versus item (content) memory. According to the associative deficit hypothesis (Naveh-Benjamin, 2000) and related theories (Hashtroudi, Johnson \& Chrosniak, 1989; Spencer \& Raz, 1995), age-related changes in spatiotemporal associative memory arise from older adults' impairment in binding together and subsequently retrieving associations among items (Allen, Morris, Stark, Fortin, \& Stark, 2015; Old \& NavehBenjamin, 2008), with spared acontextual recognition memory for the items themselves. Memory for ordinal associations is unique in that the to-be-bound items were not experienced simultaneously, so the associative link must bridge a temporal (and, in our case, spatial) gap. In a meta-analysis of aging studies on associative versus item memory, Old and Naveh-Benjamin (2008) found stronger age-related declines in temporal associative memory than other forms of associative binding. The general finding of age-impaired temporal associative memory (and associative memory in general) and spared item memory has received a great deal of empirical support from studies that probed memory for discrete laboratory stimuli (i.e., experimenter-generated lists of words or images).

Certain laboratory encoding manipulations can attenuate age-related associative memory declines, including implicit promotion of deep encoding or contextual binding (Skinner \& Fernandes, 2009; Perfect \& Dasgupta, 1997), self-referential encoding (Dulas, Newsome, \& Duarte, 2011), distinctive encoding environments (Uttl \& Graf, 
1993) and active participation in event encoding (Cheke, 2016; Hashtroudi, Parker, Luis, \& Reisen, 1989). In passive laboratory tasks, older adults may fail to spontaneously engage in adaptive encoding processes that younger adults engage automatically (e.g.

Craik, 1986). It is unclear to what degree findings of age effects on temporal associative memory generalize to memory for naturalistic experiences, in which stimuli are meaningfully (rather than arbitrarily) linked, spatiotemporal context is continuous and actively generated rather than discrete and passively perceived, sequence structure is implicit rather than strategically salient (Hintzman, 2015), and the pool of contextual information that defines each episode is comparatively rich. We reasoned that the ecologically valid conditions of our paradigm would implicitly engage active encoding processes that would attenuate the age-related decline often observed in laboratory studies. The nature of the encoding experience also allowed us to explore age effects on spatiotemporal episodic memory across naturalistic timescales.

\section{Methods}

The "Brain: The inside story" exhibit.

The "Brain: The inside story" is a traveling exhibit that was housed at the Ontario Science Centre from November 19, 2014 to March 29, 2015, with recruitment for the initial phase of this study occurring on weekends and civic holidays from February $14-$ March 29, 2015. The Ontario Science Centre is a leading attraction in Toronto with an estimated 1 million visitors annually and numerous permanent and temporary exhibits running simultaneously. It is therefore a useful venue to recruit a large sample of adults 
for research participation. Approximately 164,000 individuals visited the Brain exhibit, which contained perceptually rich educational information about the brain (e.g., sensory, memory and emotional function) aimed at a general audience.

Visitors to the "Brain: The Inside Story" exhibit were recruited by the researchers prior to entering the exhibit at a table just outside the exhibit entrance (to the left of the map in Figure 1, between the entrance (above) and exit (below) doors). Next to the table was a poster advertising a "Real-world memory experiment" for adults 18 years and older and the gift-card lottery compensation (see below). Participants were told that participation would involve following a scavenger hunt-style pamphlet guide that would not significantly alter their experience of the exhibit, and that to be eligible for compensation they would have to provide their name and email (among other information) for a follow-up questionnaire to be completed online in the future. Interested participants were given a pamphlet containing multiple-choice questions about the appearance of 17 items in the exhibit, which were selected as targets based on their distinctiveness and spacing. Participants were told that the questions were ordered according to the exhibit layout. They were instructed to find each item, examine it as they would normally, and answer the multiple-choice question (e.g. "Look at the display called "Evolving Emotions". Which of the following is NOT displayed here? (a) White rhino; (b) California seahare; (c) Green iguana; (d) Northern racoon") before moving to the next item. They were asked not to revisit items. They were instructed to fill out the pamphlet alone, and upon exiting the exhibit, to enter the time and then return the pamphlet to the researchers at the recruitment station. Notably, they were neither 
explicitly instructed to encode the target stimuli nor their order. The multiple-choice questions could be answered correctly with minimal effort, and were constructed to confirm participants' minimally sufficient attention to the target stimuli in a specified order (also dictated by the track-like physical layout of the exhibit).

Prior to entering the exhibit, participants also indicated on the pamphlet their name, sex, age, email address and entry time, and provided ratings on a 6-point Likert scale of their initial level of curiosity about the exhibit content. Upon exiting, participants recorded their exit time and rated their post-encoding curiosity about the content they encountered. Encoding duration was defined as the total time spent in the exhibit. In exchange for completing and submitting the pamphlet, participants were entered into a draw to win one of three gift cards $(\$ 200, \$ 50, \$ 50)$ to local shopping centres.

\section{Participants}

1131 participants turned in completed pamphlets at the end of the exhibit $\left(M_{\text {age }}=\right.$ 38.64, $S D=13.2)$. Of these, 1019 participants scored at least $15 / 17$ on the pamphlet questions (and thus sufficiently attended to the items) and were later invited by email to complete an online memory test for the Brain Exhibit. In exchange for completion of the online test, participants were offered a $\$ 10$ gift card to Amazon.com or iTunes. 169 successfully completed the test. This participation rate is not surprising given that the barrier to submitting a pamphlet was intentionally low (the recruitment table was immediately outside the exhibit entrance and the pamphlet was simple and unobtrusive), while still leading potential compensation, and that those who submitted 
pamphlets were a sample of adults with no pre-existing intention of participating in research. The pamphlet submission process was a way of building a pool of eligible participants rather than expected participants.

Of 169 participants who completed the online test, 9 were excluded for health reasons and one participant was excluded for having previously visited the exhibit from which the lure items were drawn (see below). We also took into consideration responses to lure trials as an empirical measure of compliance. Based on analysis of the distribution these responses, we excluded participants who correctly rejected fewer than three of sixteen (18.75\%) of the lure trials, which was deemed to reflect lack of engagement with the test, misunderstanding of instructions, or abnormally poor lure discrimination. This resulted in the exclusion of 18 participants $(11.3 \%$ of the sample; $M_{\text {age }}=44.10$ years,$S D=16.67$, range $=19-77 ; 12$ participants had 0 correct rejections, 5 had one correct rejection, and one had 2 correct rejections).

After these exclusions, 141 participants were included in the analyses (See Table 1 for descriptive statistics and Figure $\mathrm{S} 1$ for a correlation matrix including associative and item memory scores). Comparing the final 141 participants to the remainder of the 1019 participants who scored 15 or higher on the pamphlet (and thus can be said to have complied with the encoding instructions) but did complete the online test, the final participants did not differ in age $\left(M_{\text {completed }}=40.43\right.$ years, $S D=13.21 ; M_{\text {NotCompleted }}=$ 38.56 years, $S D=13.78 ; t(182)=1.5, p=.136)$ nor encoding duration $\left(M_{\text {completed }}=\right.$ 51.53 minutes, $S D=34.3 ; M_{\text {NotCompleted }}=53.04$ minutes, $S D=34.69 ; t(187)=.48, p=$ .632). The final participants had marginally higher curiosity ratings $\left(M_{\text {completed }}=5.23, S D\right.$ 
$\left.=0.86 ; \mathrm{M}_{\text {NotCompleted }}=5.06, S D=1.01 ; t(187)=2.02, p=.045\right)$ though this difference was very small $(d=.17)$. Our sample size of 141 exceeds Cohen's (1992)

recommendation of $\mathrm{N}=85$ to reliably detect a medium sized population correlation at power $=.8$ and $\mathrm{a}=.05$.

This study was approved by the research and ethics boards at the University of Toronto and Baycrest Health Sciences. All participants gave informed consent prior to participating in both phases of the experiment. 
Table 1

Descriptive statistics

Variable
Mean or count
SD Inter-quartile

range

$\begin{array}{lc}\text { Age (years) } & 40.87 \\ \begin{array}{l}\text { Gender (M/F/prefer } \\ \text { not to respond) }\end{array} & 33 / 106 / 2 \\ \begin{array}{l}\text { Handedness } \\ \text { (R/L/A) }\end{array} & 127 / 12 / 2\end{array}$

Education (years)

PHQ-9 score

Lifetime history of anxiety $(\mathrm{Y} / \mathrm{N})$
Encoding - Test delay (days)

Encoding duration (minutes)

Curiosity rating

(/6)
.17

3.21

4.02

13.89

28-49 
exhibit (one of the original 17 target items was not included because it was found to be unsuitable as a photographic visual cue). Lure stimuli were 16 pictures of items taken from the "Your Brain" exhibit at the Franklin Institute in Philadelphia, and from other similar brain exhibit pictures found online. We selected these stimuli for their feasibility as lures based on pilot data.

\section{Procedure}

Two to four months after visiting the exhibit, participants meeting inclusion criteria were invited by email to complete the online memory test. Participants completed a brief health history screening form online, including history of neurological illness (stroke, epilepsy, neurodegenerative disease or any condition requiring brain surgery, traumatic brain injury with a loss of consciousness $>15$ minutes), psychotic disorder requiring hospitalization, active drug or alcohol abuse or other major health condition affecting cognition. They also completed the Patient Health Questionnaire (PHQ-9; Kroenke, Spitzer \& Williams, 2001), a screening measure for depression, and a binary question about lifetime history of anxiety ("Have you ever suffered from significant anxiety that interfered with your functioning?"). Participants were instructed to complete the test in one sitting in a quiet room without assistance from others.

Participants completed an old/new and spatiotemporal order recognition task, conducted via the Qualtrics platform (www.qualtrics.com). Participants were shown pairs of pictures and had to indicate whether or not the item pairs were shown in the same order as encountered in the exhibit (i.e., intact or re-ordered) or if either of the items was not from the Brain Exhibit (i.e., new). There were 16 intact, 16 re-ordered, 
and 16 new pairs in total. Participants clicked either 'Correct order', 'Wrong order' or 'New'. Participants were instructed to respond 'New' if either picture was not at the brain exhibit, but both pictures were in fact new on these trials. In addition, we varied the spatiotemporal distance of old (intact and re-ordered) pairs, such that half of the old pairs consisted of adjacent target items in the exhibit (i.e., near pairs) and half consisted of items with 2 intervening target items between them (i.e., far pairs) (see Figure 1). For both old and new pairs, individual items appeared in multiple pairs, but each pair appeared in a given order only once. It is worth noting that in an fMRI study of spatial/temporal order memory with a similar design, where individual items were repeated in multiple unique combinations, task-critical medial temporal and prefrontal brain regions were not sensitive to individual item repetitions (Ekstrom, Copara, Isham, Wang, \& Yonelinas, 2011). Accordingly, tasks of this nature are thought to tap into processing of item associations, which were trial-unique, over and above the constituent items. 

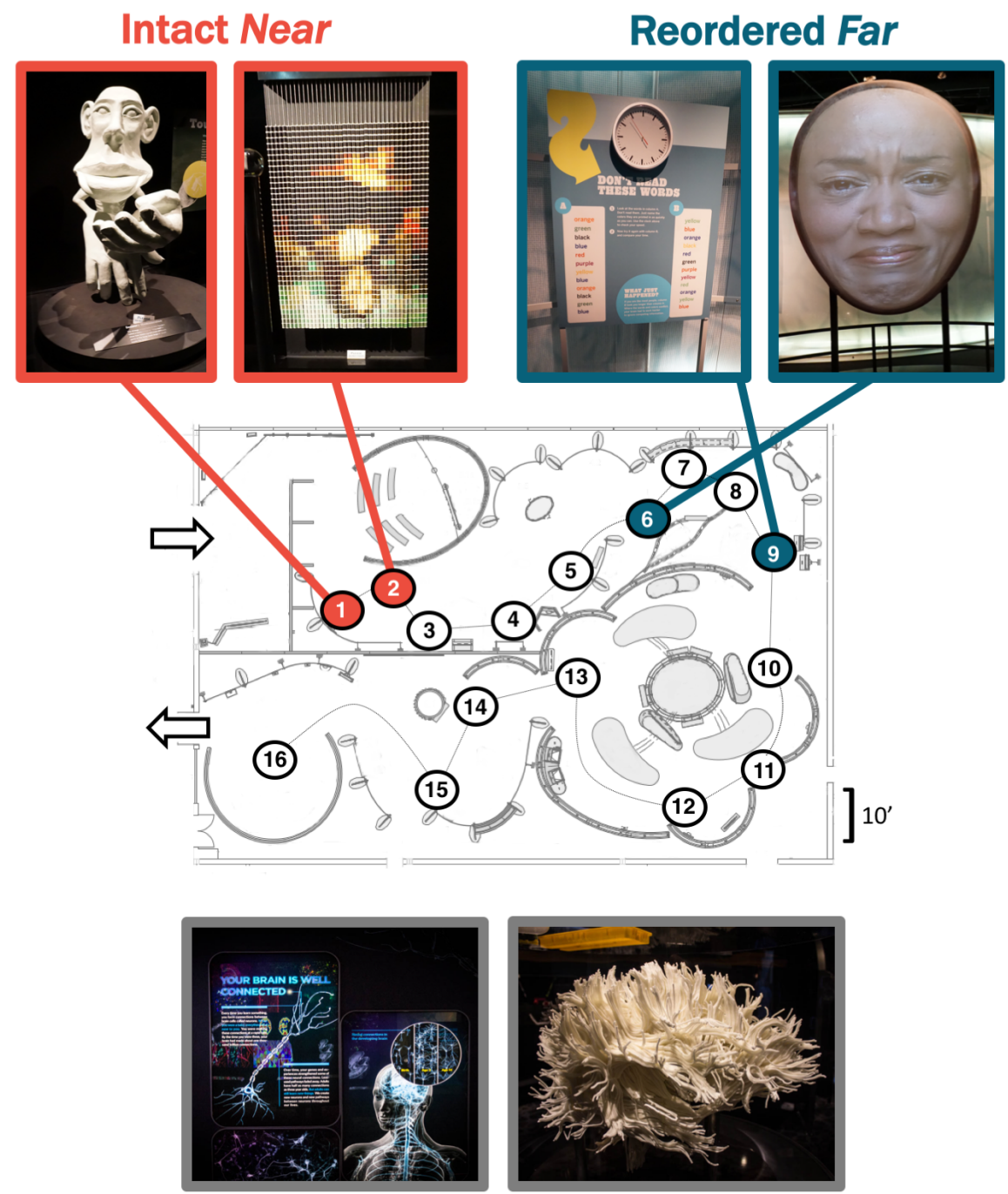

New

Figure 1. Diagram outlining three example stimulus pairs from the recognition memory test. The map depicts the structure of the exhibit and numbered red circles depict the approximate location of each target item and the order in which they were viewed. (A) Near (adjacent) and intact (in the correct order, from left to right); (B) Far (two intervening target items) and re-ordered (in the wrong order from left to right); $(C)$ Lure pair (new pictures drawn from a different brain museum exhibit). All photo stimuli were the same size; images are cropped and re-sized here for display purposes.

\section{Data analysis}

Before extracting estimates of associative and item memory and investigating their relationship to aging, we characterized performance on our old/new and spatiotemporal 
order memory test by modelling performance as a function of age (continuous betweensubjects regressor) and our memory cue manipulations (categorical within-subjects factors). We fit the data with linear mixed effects models using the Imer() function within the Ime4 (Bates, Maechler, Bolkder, \& Walker, 2015) and ImerTest (Kuznetsova, Brockhoff, \& Christensen, 2015) packages in the R programming language, first at the highest level of cue pair validity (i.e., intact/reordered/new) and then within 'old' pairs (intact/reordered and near/far). In both models, we averaged performance over trials of each type within participants. Participants were modelled with a random intercept and significance tests using the Satterthwaite approximation for degrees of freedom, which produces relatively conservative Type 1 error rates (Luke, 2017). We used a similar model to investigate the interaction between age and error types. Age was meancentred in all models. Post-hoc Welch's t-tests (degrees of freedom rounded to the nearest whole number) were Bonferroni-corrected for multiple comparisons. We report Cohen's d estimates of effect size. When measuring associations between task performance and age, we computed Kendall's rank correlation (tau) in addition to Pearson's $r$ when score distributions were highly non-normal due to ties at floor or ceiling. All significance tests were two-tailed.

We adapted the logic of process dissociation (Jacoby, 1991) to calculate independent estimates of associative and item memory under a signal detection framework (for similar approaches, see: Cohn, Emrich, \& Moscovitch, 2008; Troyer, D’Souza, Vandermorris, \& Murphy, 2011; Troyer et al., 2012; Wolk, Signoff, \& DeKosky, 2008; Yonelinas, Regehr, \& Jacoby, 1995). Specifically, we computed associative 
memory (associative d') as the standardized proportion of hits to intact and reordered pairs minus the standardized proportion of order errors (see Equation 1), thereby isolating accurate spatiotemporal context memory over and above item recognition.

$Z\left(\frac{P\left(\text { 'intact' }^{\prime} \mid \text { intact }\right)+P\left(\text { 'reordered }^{\prime} \mid \text { reordered }\right)}{2}\right)-Z\left(\frac{P\left(\text { 'intact' }^{\prime} \mid \text { reordered }\right)+P\left(\text { 'reordered' }^{\prime} \mid \text { intact }\right)}{2}\right)$

Item memory (item d') was calculated as a standard old/new discrimination score.

$$
Z(P(\text { 'intact' I intact }))-Z(P(\text { 'intact' I new }))
$$

Hit rates of $100 \%$ and false alarm rates of $0 \%$ were corrected by artificially adding half of one miss or false alarm, respectively ( $P_{\text {hit }}=1$ corrected to $1-1 /(2 \mathrm{~N})$ and $P_{F A}=1$ corrected to $1 /(2 N)$, where $N$ is the number of eligible trials) (Macmillan \& Creelman, 2005).

We note that in previous studies where estimates of item and associative memory or recollection and familiarity were extracted from a single test (using 'exclusion test' conditions from the process dissociation procedure (Jacoby, 1991; Troyer et al., 2011; Wolk et al., 2008)), there are typically two response options (e.g., 'old' and 'new'). Participants are instructed to respond 'old' selectively to old stimuli presented in the correct context ('intact', in the present study) and to respond 'new' to both totally new test stimuli and old test stimuli presented in the incorrect context; therefore, hits only include correct recognition based on item and context. The inclusion of third response option in the present study ('reordered') allows one to dissociate correct rejections based on context violations ('reordered' I reordered) versus lures ('new' I new), as well as more inclusive operationalizations of misses ('new' I intact or reordered) and false alarms ('intact' or 'reordered' I new), but raises the question of how to theoretically 
integrate 'reordered' hits into previously established formulas. We reasoned that, from the perspective of associative memory, intact and reordered hits similarly signal correct recognition of the items and their spatiotemporal context (and the converse, order errors, signal recognition in the absence of context). Conversely, we used a standard measure of item memory consistent with previous studies (Troyer et al., 2011; Troyer et al., 2012; Wolk et al., 2008), based on a dual-process signal detection model (Yonelinas et al., 1995), including only 'intact' responses to intact pairs (hits) and new pairs (false alarms). We reasoned that from the perspective of item memory, 'intact' and 'reordered' responses do not equally signal familiarity for the items irrespective of their spatiotemporal context. Accordingly, of false alarms to new trials, 'intact' responses were more prevalent than 'reordered' responses $\left(M_{\text {intact }}=12.41 \%\right.$ of new trials, $M_{\text {reordered }}$ $=7.18 \% ; t(140)=4.498, p<.001)$. We report results using alternative formulas in the Supplementary Material (Figure S2; Associative $2=Z(P($ 'intact' I intact) $)-Z(P($ 'intact' I reordered)), consistent with previous studies; Item $2=Z(P$ ('intact' or 'reordered'। intact or reordered $))-Z(P($ ('intact' or 'reordered'l new $))$, isolating old/new discrimination irrespective of old pair order; Item $3=Z(P$ (order errors $))-Z(P$ ('intact' or 'reordered' I new)), isolating old/new discrimination in the absence of associative memory). The main findings held under all operationalizations of item and associative memory. A broader examination of test responses is considered in our error type analyses.

To investigate the unique effect of aging and potential contributions from other variables on memory, we conducted separate multiple regressions upon associative and item memory estimates, modelling age as our regressor of interest in addition to 
demographic and health information (sex, handedness, years of education, depression [PHQ-9 score] and anxiety) and extraneous memory-related factors (remoteness, encoding duration and pre-encoding curiosity ratings) (see Table 1). As encoding duration, PHQ-9 scores, education, and curiosity were significantly skewed, these scores were square root (education) or log (encoding duration, PHQ-9, and curiosity) transformed for the purposes of regression analysis.

We also recorded response times (RT). Our main purpose in assessing RT was to assess task compliance and confirm validity of the responses, given that testing was unsupervised. We expected to find that reaction times would be sensitive to our manipulation of cue validity (old vs. new), order (intact vs. re-ordered) and distance (near vs. far). These findings would further support our use of the internet testing platform. Analyses were restricted to correct trials.

Mirroring our analyses of recognition memory performance (see Data Analysis and Results), we first modelled participant-wise median RT as a function of pair type (intact, re-ordered or new; within-subjects factor) and age (between-subjects regressor). There were main effects of pair type $(F(2,274)=22.66, p<.001)$, where both new and re-ordered trials elicited faster responses than intact trials $(t(138)=5.62, p<.001, d=$ .48 , and $t(138)=6.57, p<.001, d=.56$, respectively $)$ and age $(F(1,137)=51.02, p<$ $.001)$, whereby response times increased with age $(r=.52, p<.001)$. The interaction between age and pair type was not significant $(F(2,274)=2.35, p=.097)$ (see Table 2).

Next, we investigated RT within the old pairs as a function of age, order (intact vs. re-ordered) and distance (near vs. far). Four participants had zero correct trials in a 
given cell - they were included in the model but not in the post-hoc t-tests. In addition to main effects of age $(F(1,133)=39.01, p<.001)$ and $\operatorname{order}(F(1,399)=59.05, p<.001)$ as above, there was a significant interaction between age and order $(F(1,399)=7.79, p$ $=.006)$ such that the age-related increase in RT was slightly greater for intact $(r=.41$, $p<.001)$ versus reordered trials $(r=.37, p<.001)$. Furthermore, there was a main effect of distance $(F(1,399)=63.63, p<.001)$ where responses were faster for far than for near pairs $(t(134)=8.91, p<.001, d=.33)$, extending previous findings. There was also an interaction between order and distance $(F(1,399)=7.47, p=.007)$. Far reordered pairs elicited the fastest responses (all p's $<.001$ uncorrected) and near intact pairs elicited the slowest responses (all p's $<.001$ uncorrected). That participants' response times were sensitive to our context manipulation and exhibited expected effects of item distance supports the contention that participants completed the online test according to instructions, with high compliance, and that their responses were valid at the item level.

Table 2

Mean $(M)$ and standard deviation $(S D)$ of participant-wise median response times for each pair type.

\begin{tabular}{lcc}
\hline \multicolumn{1}{c}{ Pair type } & $M(s)$ & $S D(s)$ \\
\hline Intact near & 9.73 & 5.31 \\
Intact far & 7.34 & 3.94 \\
Reordered near & 7.37 & 3.77 \\
Reordered far & 6.10 & 3.40 \\
New & 6.63 & 3.96 \\
\hline
\end{tabular}




\section{Results}

We first characterized performance on our recognition memory test at the highest level by modelling accuracy as a function of age and the three main pair types (intact, reordered and new). There were main effects of age $(F(1,139)=21.75, p<.001)$, with overall accuracy decreasing as a function of age $(r=-.37, p<.001)$, and pair type $(F(2,278)=75.03, p<.001)$, where new pairs elicited greater accuracy than both intact $(t(140)=7.43, p<.001, d=.63)$ and reordered pairs $(t(140)=10.37, p<.001, d=.87)$, and intact trials elicited greater accuracy than reordered trials $(t(140)=4.36, p<.001, d$ $=.37)$. Furthermore, there was an interaction between pair type and age $(F(2,278)=$ $9.38, p<.001)$, such that increasing age predicted lower accuracy on new $(r=-.39, p<$ $.001)$ and re-ordered $(r=-.25, p=.003)$, but not intact $(r=-.14, p=.094)$ pairs (see Figure 2).

Within the old pairs, we modelled accuracy as a function of age and pair order (intact vs. reordered) and pair distance (near vs. far). In keeping with the above results, there were significant main effects of age $(F(1,139)=7.38, p=.007$,$) and pair order$ $(F(1,417)=17.76, p<.001)$. There was also a main effect of distance $(F(1,417)=$ $45.12, p<.001)$, whereby far pairs elicited greater accuracy than near pairs $(t(140)=$ $7.21, p<.001, d=.61)$, and no interaction between distance and age $(F(1,417)=.22, p$ $=.64)$, consistent with previous findings that the benefit of greater inter-item distance to accurate contextual retrieval is age-invariant (Fabiani \& Friedman, 1997; Perlmutter et al., 1981; Tolentino et al., 2012). There were no interactions between pair order and age 
$(F(1,417)=2.37, p=.12)$, pair order and distance $(F(1,417)=.62, p=.43)$, nor was there a three-way interaction $(F(1,417)=2.73, p=.099)$ (see Figure 2).
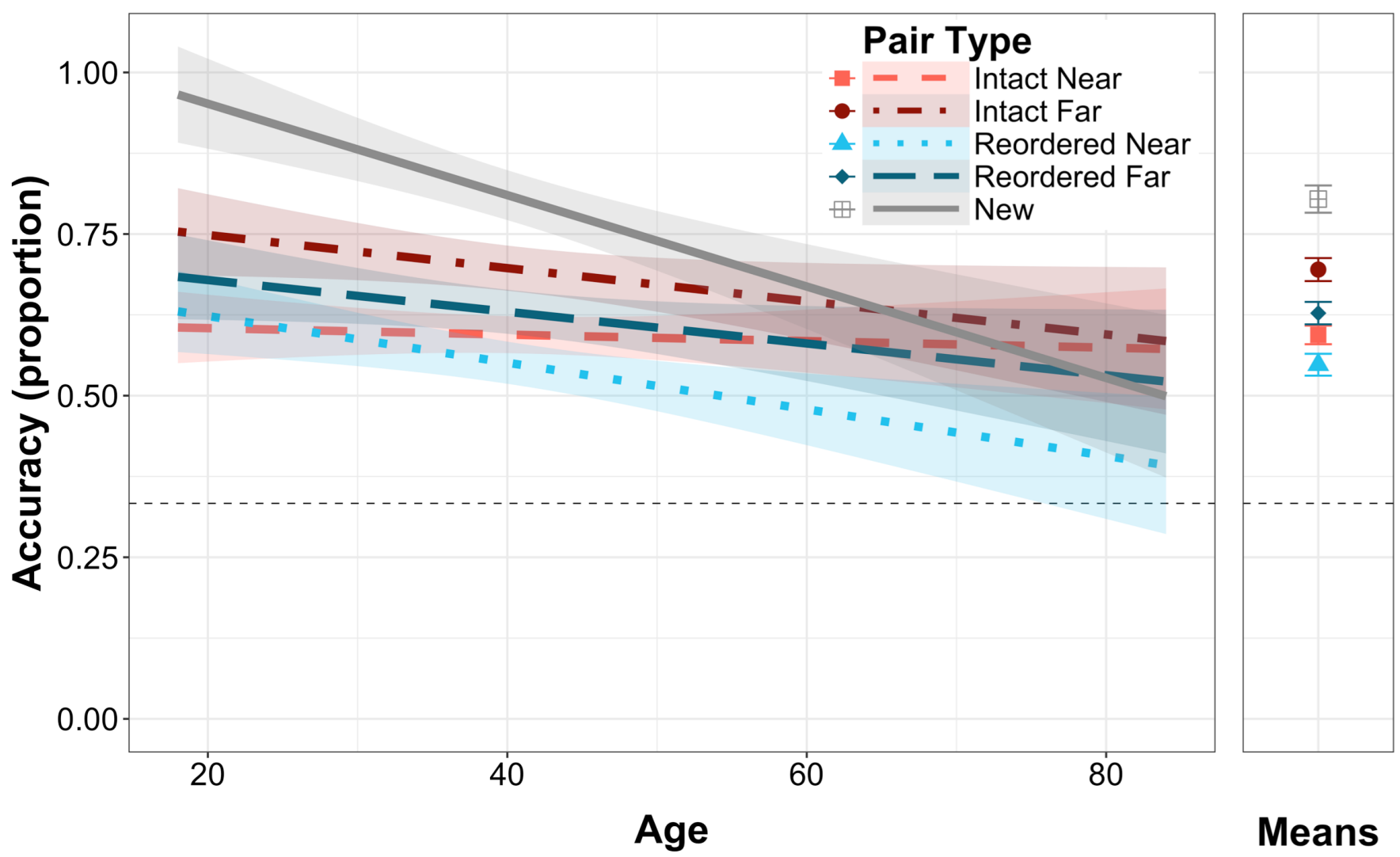

Means

Figure 2: Performance on each trial type. Left: correlation of performance on each trial type with age (shaded region displays 95\% confidence interval around linear model trend lines). Right: circles display whole-sample means and lines represent standard errors of the means. The dotted black line (accuracy $=0.33$ ) signifies chance performance.

Modelling the effect of age on associative and item memory using multiple regressions, age was not significantly associated with associative memory $(\beta=-.16, t=$ $-1.55, p=.124$, semi-partial $r=-.14)$, though there was a trend towards a reduction with 
age. There was a non-significant trend towards better associative memory with greater total encoding duration $(\beta=.18, \mathrm{t}=1.90, p=.06$, semi-partial $r=.17)$, consistent with previous findings of encoding duration at the item level (Vilberg \& Rugg, 2009). None of the other predictors (sex, handedness, years of education, depression, anxiety, remoteness, encoding duration and pre-encoding curiosity ratings) were significant (all $p$ 's $>$.25). The variance inflation factors (VIF) for age and total encoding duration were 1.29 and 1.17, respectively, ruling out potential issues of collinearity (VIF for all other variables < 1.4).

For item memory, age was a significant predictor of performance $(\beta=-.34, \mathrm{t}=-$ 3.48, $p=.001$, semi-partial $r=-.29$ ). Age had a stronger than expected negative association with item memory. Years of education was positively associated with item memory $(\beta=.22, \mathrm{t}=2.51, p=.014$, semi-partial $r=.21)$. No other variables predicted item memory performance (all p's $>.15$ ).

Scatterplots showing the zero-order correlations between age and associative memory versus item memory are presented in Figure 3. To compare associative and item memory, we modelled memory type (associative vs. item) as a within-subjects factor and age as a continuous predictor of performance. We found main effects of age $(F(1,139)=9.48, p=.003)$, wherein overall memory performance decreased with age $(r$ $=-.25, p=.003)$, and a main effect of memory type $(F(1,139)=311.54, p<.001)$, wherein item memory $(M=1.73, S D=.89)$ was greater than associative memory $(M=$ $.83, S D=.66)$ overall $(t(140)=17.03, p<.001, d=1.43)$, which is unsurprising given that item memory is thought to be less effortful (Jacoby, 1991; Old \& Naveh-Benjamin, 
2008). There was a significant interaction between memory type and age $(F(1,139)=$ $11.44, p<.001)$, such that age was negatively associated with item memory $(r=-.30$, $\left.R^{2}=.09, p<.001\right)$ but not associative memory $\left(r=-.14, R^{2}=.02, p=.09\right)$ and the coefficients were significantly different from each other $(Z=2.53, p=.012$; ; Steiger, 1980). Removal of the oldest participant (84 years old) did not significantly alter the results of the aforementioned analyses (with participant removed: Associative $r=-.13$, $p=.124 ;$ Item $r=-.29, p<.001)$, and alternative associative and item memory formulas produced similar coefficients (see Figure S2). Given that associative memory was poorer than item memory overall, we assessed the possibility that it was at chance, which would artificially eliminate the possibility of observing an age-related decline. We tested whether scores at the low (18-35 years old) and high extremes of the age sample (60+ years old) were significantly above zero. Associative memory in both the youngest $(M=.93, S D=.65 ; t(50)=10.16, p<.001)$ and oldest adults $(M=.73, S D=.74 ; t(17)=$ $4.23, p<.001$ ) was significantly above chance (see Figure S3). 

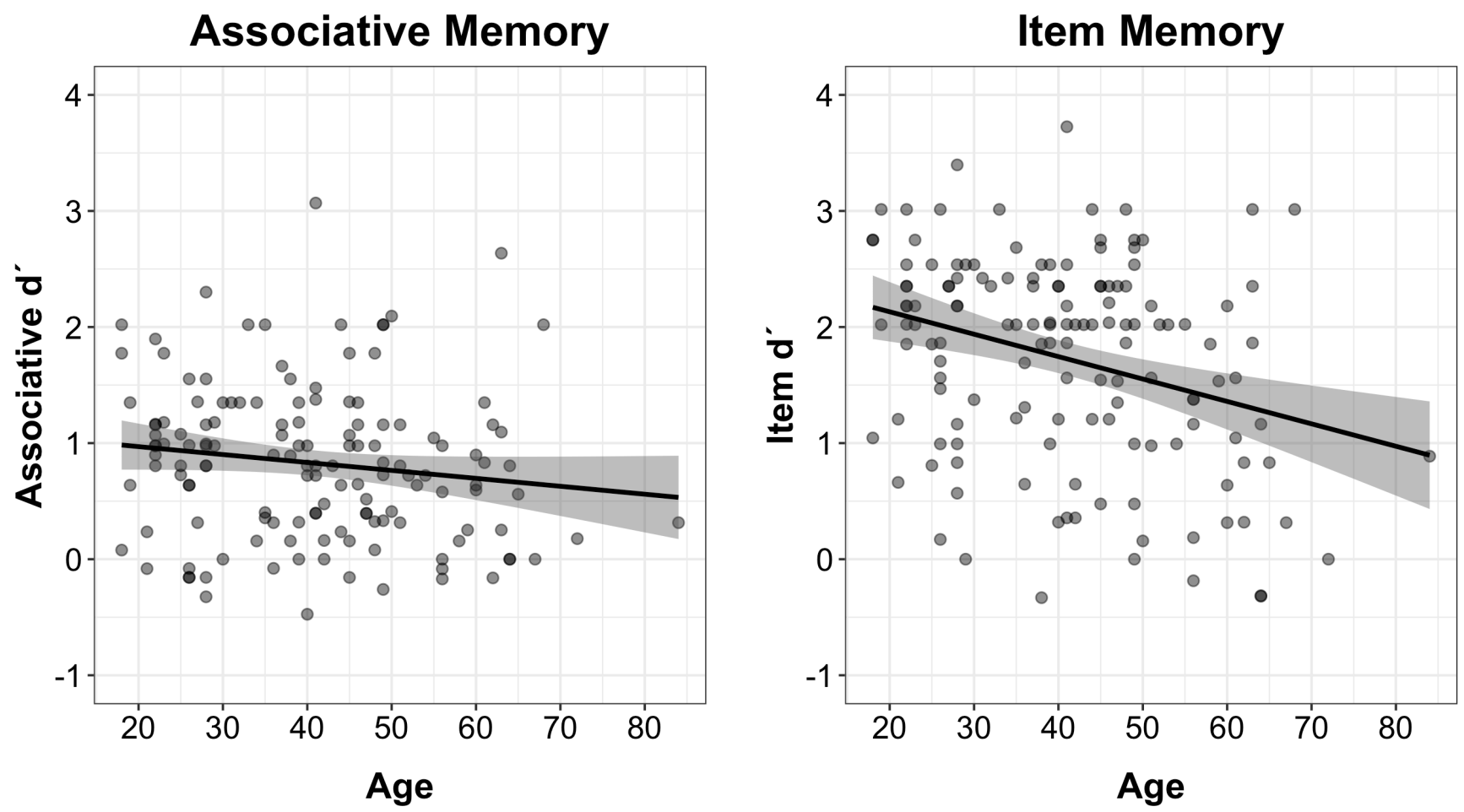

Figure 3: Scatterplots displaying zero-order correlations between age and associative (left; $r=-.143, p=.09$ ) and item (right; $r=-.302, p<.001$ ) memory. Dots represent individual participants. Shaded region displays $95 \%$ confidence interval around a linear model trend line.

To further unpack the nature of memory decisions driving the observed age effects on item vs. associative memory, we investigated the types of errors contributing to these scores. We operationally defined three broad classes of errors: order errors ('reordered' responses to intact pairs and 'intact' responses to reordered pairs, isolating spatiotemporal context error in the presence of accurate old/new recognition), misses ('new' responses to old [intact and re-ordered] pairs) and false alarms (old ['intact' and 'reordered'] responses to lures). Modelling error type as a within-subjects categorical regressor and age as a continuous regressor yielded main effects of age $(F(1,139)=$ $25.78, p<.001)$ and error type $(F(2,278)=97.50, p<.001)$, with order errors being 
more common than both misses $(t(140)=19.45, p<.001, d=1.64)$ and false alarms $(t(140)=5.90, p<.001, d=.5)$, and false alarms being more common than misses $(t(140)=5.92, p<.001, d=.5)$. Furthermore, there was a significant interaction between age and error type $(F(2,278)=15.94, p<.001)$, with age positively associated with increasing proportion of misses $(r=.27, p=.001$; tau $=.16, p=.011)$ and false alarms $(r=.39, p<.001 ;$ tau $=.26, p<.001)$ but not order errors $(r=.04, p=.602)$ (see Figure 4).

To reconcile our observation of age-related decline in performance on reordered trials with the contrasting stability in associative memory and order errors, we ran exploratory correlations between age and the two types of errors one can make on reordered trials: false alarms ('intact' I reordered) and misses ('new' I reordered). We found that aging was associated with an increase in misses $(r=.28, p<.001 ; \operatorname{tau}=.18$, $p=.005)$ but not false alarms $(r=.09, p=.305$; tau $=.08, p=.18)$ on reordered trials, although these correlations were not significantly different from each other $(Z=1.65, p$ $=.099)$. 

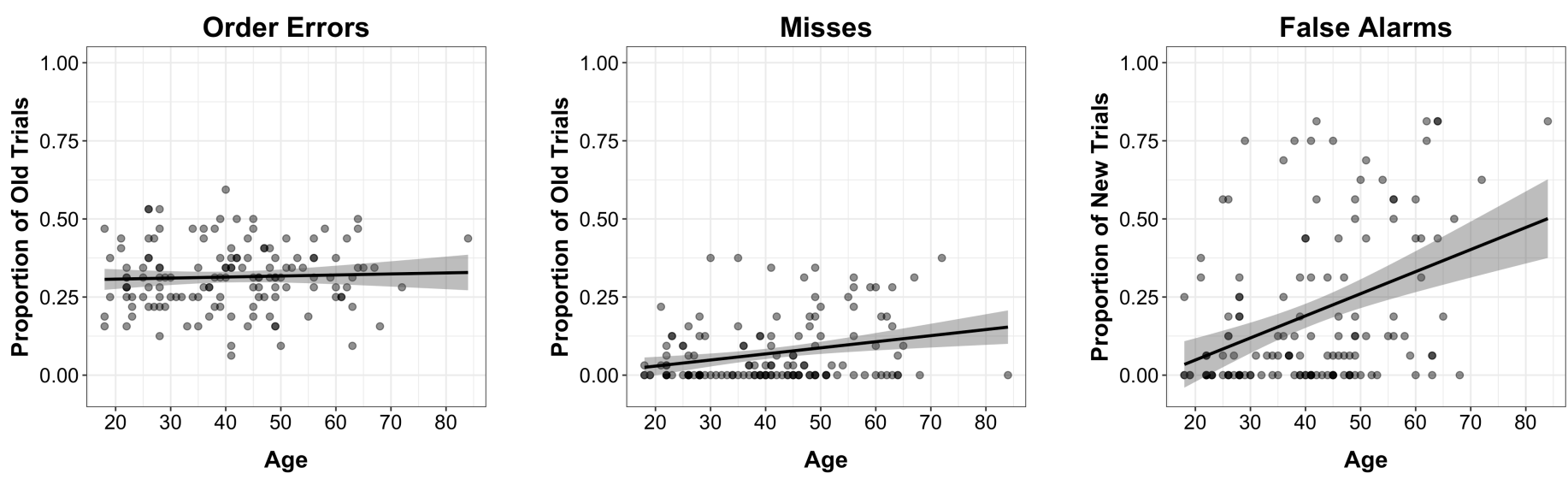

Figure 4: Correlations between age and error types (Order errors: $r=.044, p=.6$; Misses: $r=.27, p=.001$, tau $=.16, p=.011$; False Alarms: $r=.39, p<.001$, tau $=.26, p$ $<.001)$. Each error type is calculated as a proportion of the number of appropriate trials. Dots represent individual. Shaded region displays 95\% confidence interval around a linear model trend line.

While our continuous age sample provides a unique window into changes in distinct retrieval processes across the lifespan, most of the literature motivating this research models age as categorical younger and older groups. Thus, to facilitate interpretation and comparison with related studies, we analyzed and visualized associative and item memory scores, as well as error types, using canonical younger (18-35 years) and older (60+ years) groups. Consistent with the above findings, there was an interaction between age group and memory type, wherein item discrimination was significantly lower in older adults ( $d=.84 ; 44.44 \%$ lower than younger adults) but associative memory was not ( $d=.26 ; 20.98 \%$ lower than younger adults; Figure S3). There was also an interaction between age and error type, wherein older adults exhibited a two-fold increase in misses and a three-fold increase in false alarms relative to younger adults, with no difference in order errors (Figure S4). 


\section{Discussion}

We attempted to enhance concordance between laboratory and naturalistic methods of memory assessment using a novel, one-shot, real-world episode (exploring a museum exhibit) prospectively for subsequent memory testing. Spatiotemporal associative memory and item memory were assessed with a recognition memory paradigm using target and lure photographs, allowing us to dissociate, in a large lifespan sample, age effects on item discrimination and spatiotemporal order retrieval.

Contrary to an extensive literature documenting age-related deficits in associative memory (Old \& Naveh-Benjamin, 2008), there was no significant effect of age on spatiotemporal associative memory using adaptations of standard processdissociation measures. Accordingly, there was no age-related increase in order errors, and the implicit effect of inter-item distance on order memory accuracy and response times did not interact with age. Conversely, we found a significant reduction in item memory over the lifespan driven primarily by a marked increase in susceptibility to false alarm to novel lures. Lure susceptibility could not be accounted for by more liberal response criteria in older adults because aging also was associated with increased misses. While previous studies have reported age-related reductions in temporal order memory alongside item memory reductions (Newman et al., 2001) and false alarm increases (Daum, Gräber, Schugens, \& Mayes, 1996), we are not aware of any prior report of a greater age-related reduction in item versus associative memory. This dissociation challenges the notion that age-related declines in memory for content, 
where observed, are necessarily driven by impairments in memory for context (Dumas \& Hartman, 2003).

\section{Spatiotemporal associative memory}

The attenuated age effects on associative memory in the present study may be attributable to encoding factors that promote subsequent associative memory, such as active exploration and goal-directed movement (Kaplan et al., 2012; Plancher et al., 2012; Voss et al., 2011). In exploration of environmental-scale spaces, spatial and temporal relationships are actively constructed via self-motion rather than passively perceived (Buzsáki \& Llinás, 2017), enhancing relational memory (Wang and Simons, 1999) due to contributions of vestibular, kinesthetic and proprioceptive cues (Taube, Valerio, \& Yoder, 2013). Active exploration may automatically recruit hippocampally mediated associative processing circuits and strategies that younger but not older adults typically engage spontaneously in impoverished encoding conditions. For instance, passive transport in a wheelchair disrupts the formation of spatial representations in older adults relative to younger adults but both groups perform similarly when actively navigating (Adamo, Briceño, Sindone, Alexander, \& Moffat, 2012; Allen, Kirasic, Rashotte, and Haun, 2004). A review of earlier spatial memory literature found that older adults perform similar to younger adults in real-life but not laboratory environments due to greater distinctiveness of contexts in which items are embedded in real life environments (Uttl and Graf, 1993). 
The exhibit items were not only linked by participants' physical locomotion but also by semantic similarity, in contrast to typical associative memory studies using intentionally arbitrary stimuli. Semantic relatedness among studied items has been shown to facilitate the formation of temporal contextual associations during encoding by encouraging reactivation of previously encountered related items (Tzeng \& Cotton, 1980). One might also suspect that prior knowledge about museum exhibits could lend schematic support to associative memory, for older adults in particular (Castel, 2005). However, such knowledge would neither bear on the idiosyncratic details of these particular items nor their sequence, and therefore would not have systematically altered temporal order judgements in this study.

Intentional encoding of associations has been found to benefit younger adults more than older adults and thereby increase the age-related gap in associative memory (Old \& Naveh-Benjamin, 2008). In our study, the encoding instructions explicitly cued attention towards perceptual features of the exhibit items, but not their order. It could be argued that this difference explains the observed age-related decline in item memory and stability in item memory. However, even in previous studies where associative memory was incidentally encoded and item memory was intentionally encoded, associative memory still declined with age as much or more than item memory (Old \& Naveh-Benjamin, 2008; Schmitter-Edgecombe \& Simpson, 2001). Regarding temporal associations in particular, evidence for effects of intentional versus incidental encoding has been debated (Michon \& Jackson, 1984; Naveh-Benjamin, 1990; Zacks, Hasher, Alba, Sanft, \& Rose, 1984), with intentional encoding sometimes boosting temporal 
order memory in both younger and older adults (Schmitter-Edgecombe \& Simpson, 2001) and sometimes in neither (Kausler, Lichty, \& Davis, 1985), and with age effects typically observed either way (Kausler \& Wiley, 1990). Therefore, based on the literature, one would expect to observe an age-related decline in temporal associative memory based on incidental encoding alone.

At test, naturalistic photographs of the items (including their immediate local contexts) were likely potent retrieval cues, triggering implicit context reinstatement and providing retrieval support that may also have conferred greater benefits to older than to younger adults (Cohn, Emrich \& Moscovitch, 2008). In previous studies, context reinstatement has been found to improve recognition performance in older adults as much or more than in younger adults, in some cases ameliorating the age gap altogether (Craik \& Schloerscheidt, 2011; Naveh-Benjamin \& Craik, 1995; Robin \& Moscovitch, 2017). Consistent with this interpretation, we found that performance on intact pairs was stable with age but performance on reordered pairs - where the constituent items were valid cues but the spatiotemporal context was violated declined. The age-related decline in performance on reordered trials was driven by increased misses, but not order errors, and is therefore not inconsistent with the main finding of preserved spatiotemporal associative recognition memory in older adults. This finding could be related to reduced flexibility in spatiotemporal relational representations with age (Etchamendy, Konishi, Pike, Marighetto, \& Bohbot, 2012), such that aging spares recognition of intact contextual cues but impairs flexible reconstruction of these cues, leading older adults to perceive old-but-reconfigured elements as new. 
We would predict steeper age-related reductions in spatiotemporal contextual memory for the exhibit had we used a task that placed higher demands than recognition on self-initiated processing and strategic retrieval (Cohn et al., 2008; Craik, 1986), for instance free recall (e.g. Dumas \& Hartman, 2003; Levine et al., 2002) or serial order reconstruction (e.g. Kausler \& Wiley, 1990; Schmitter-Edgecombe \& Simpson, 2001), or with speeded rather than self-paced response windows (Roberts, Ly, Murray, \& Yassa, 2014). Given that we did not implement an immediate test, another possibility is that forgetting over the months-long delay interval rendered our test less sensitive to age differences in associative memory that may have been apparent at shorter delays. We cannot rule out this possibility, although prior studies suggest that temporal order memory for both laboratory stimuli and naturalistic actions performed in the laboratory decays at similar rate in younger and older adults (Kausler \& Wiley, 1990; Perlmutter et al., 1981).

Item memory

The age-related increase in lure susceptibility is consistent with recent crossspecies evidence that aging affects discrimination of familiar from novel information due to changes in medial temporal lobe circuits responsible for high-level object representation, especially when familiar and novel items have high feature overlap (Burke et al., 2011; Reagh et al., 2016; Ryan et al., 2012; Yeung, Ryan, Cowell, \& Barense, 2013; Johnson et al., 2017). Combined with age-related source monitoring impairments, this may produce elevated false recognition (Hashtroudi, Johnson \& 
Chrosniak, 1989; Schacter, Koutstaal, Johnson, Gross, \& Angell, 1997). This view is contrasted with the notion that age-related changes are due to a weakening of memory traces.

Previous studies have shown that false recognition of events can be induced in older adults, for instance by watching naturalistic videos and then reviewing plausible lure photos that were not in fact in the videos (Schacter et al., 1997). In the present study, we found age-related increases in lure susceptibility without any manipulation to promote it, alongside largely preserved associative memory for valid memoranda. Given that both valid memoranda and lures in the present study were naturalistic photos depicting brain-themed museum exhibit items, it is likely that perceptual and conceptual similarity between targets and lures, and corresponding over-reliance on gist information during discrimination, contributed to the increase in false recognition with age (Koutstaal \& Schacter, 1997; Pidgeon \& Morcom, 2014). Our findings are more consistent with models of aging emphasizing mnemonic discrimination impairments than with the associative deficit hypothesis (Naveh-Benjamin, 2000), but more work is necessary to adjudicate between these views.

\section{Task compliance}

Regarding our paradigm, as the memory test was conducted online and was unsupervised (see Armson, Abdi, \& Levine (2016), for a similar approach), it is crucial to determine that participants responded in a valid and reliable manner. We ruled out inattentive or random responding by excluding those who performed very poorly on 
discrimination of new items. Sensitivity in participants' response times to our old/new and context manipulation helped to establish task compliance. Furthermore, we replicated established effects of context reinstatement, in that performance was greater on intact compared to reordered trials, and age-invariant sensitivity to inter-item distance (i.e., better performance and faster responses on far than on near pairs) in line with previous studies (Fabiani \& Friedman, 1997; Perlmutter et al., 1981; St. Jacques et al., 2008; Tolentino et al., 2012; but see Campbell, Trelle, \& Hasher, 2014). These results build upon recent work using large-scale real-world encoding paradigms to explore spatiotemporal organization in episodic memories (Griffiths et al., 2016; Nielsen et al., 2015; St. Jacques et al., 2008). We extend the results of these studies, which tested memory for events in familiar environments at shorter delays, to a cognitive aging context and show some preservation of spatiotemporal order memory for the elements of a one-shot episode at a longer delay and finer spatiotemporal scale than previously reported. Even on the most difficult adjacent re-ordered pairs, thought to require finegrained episodic reconstruction (Curran \& Friedman, 2003; St. Jacques et al., 2008), performance was well above chance (whole-sample mean $=.55$; chance $=.33$ ).

\section{Limitations}

The influence of naturalistic encoding processes per se on the present findings remains speculative; inclusion of a comparable and time-matched laboratory assessment would help to establish the specificity of the observed effects to naturalistic events. Furthermore, unlike previous studies, we could not dissociate spatial and 
temporal aspects of memory organization (e.g. Allen et al., 2015; St. Jacques et al., 2008) - accurate associative retrieval could involve retracing the egocentric trajectory of target items and/or by remembering their allocentric spatial locations in a map-like representation. Nevertheless, the architectural structure of museum exhibits makes them useful in particular for testing naturalistic spatiotemporal memory and facilitating translation between rodent models and human research (Zisch, Gage, \& Spiers, 2014). It is also not clear to what degree order memory performance in the present task generalizes to other forms of associative memory (see Old \& Naveh-Benjamin, 2008) or mnemonic context more broadly (Stark, Reagh, Yassa, \& Stark, 2017). Nor is it clear to what degree successful associative memory involved subjective recollection of the encoding episode. Indeed, recent work suggests that ordinal associative memory (at short delays) may not depend on recollection (Brunec, Ozubko, Barense, \& Moscovitch, 2017). Future work is necessary to determine the strategies and phenomenological states accompanying spatiotemporal context retrieval and how they change with age.

Another potential limitation concerns the representativeness of the sample in the present study. Although the sample and recruitment method are different from the typical aging study recruiting younger adults from a pool of undergraduates, usually psychology students, and older adults from research participant databases, the Ontario Science Centre is a highly generic and popular attraction in Toronto. There is no reason to suspect that recruitment from such an attraction is less representative of the general population than typical recruitment methods. Indeed, several high impact memory studies have used museum exhibits in naturalistic encoding paradigms (e.g. Aggleton \& 
Waskett, 1999; Henkel, 2013; St. Jacques, Olm, \& Schacter, 2013; St. Jacques \& Schacter, 2013), and prior visits to museum exhibits are often used as cues in autobiographical memory studies (e.g. Robin \& Moscovitch, 2014; Hassabis et al., 2007) for their assumed universality.

A second concern about the present sample is that it lacks representation at the older end of the age spectrum. We would expect to observe a decline in spatiotemporal associative memory at the higher end of the age spectrum had our sample included more participants in that range. However, one would expect continued decline in item memory as well. Moreover, the age distribution in our sample is comparable to that of many laboratory studies reporting age-related declines in spatiotemporal memory and associative memory (see Old \& Naveh-Benjamin, 2008). Finally, continuous lifespan investigations of associative versus item memory (Bender, Naveh-Benjamin, \& Raz, 2010) and corresponding brain atrophy (Raz et al., 2005) found incremental changes beginning in early adulthood and accelerating in middle rather than late age. Therefore, to the extent that significant age-related declines in temporal associative memory are expected based on the extant literature, they should be detectable in the present sample. Nonetheless, we cannot make inferences about memory changes in older age (i.e., 70 years and older) based on the present sample.

Finally, the effect of aging on associative memory could in principle be artificially truncated by the lower performance of younger adults in associative versus item memory. This would affect comparisons of the associative and item memory slopes. Therefore, the magnitude of this difference should be interpreted with caution. Yet 
despite the long delay and fine grain at which ordinal discrimination was tested, older adults' performance was well above chance (associative $d^{\prime}=.73$ in participants $60+$ years old). The null effect of age on associative memory cannot be explained by the lower overall performance on associative memory, unless there was a feature of the task that selectively reduced younger adults' performance. Moreover, the dissociation between age effects on associative and item memory is supported by multiple lines of evidence: there was no age-related increase in order errors, where the distribution is well off of floor and ceiling, and the influence of inter-item distance on ordinal discrimination did not interact with age.

\section{Conclusions}

Laboratory research has demonstrated that spatiotemporal context is a fundamental component of episodic memory (Ranganath \& Hsieh, 2016; Tulving, 1972; Howard \& Kahana, 2002), and it is assumed that this contextual detail is a core feature of our personal memories from months and years ago. However, due to methodological limitations, little is known about memory for the order of real-world experiences over delays typical of everyday episodic memory, and how this capacity changes with age. The present study provides further validation for assessing memory using verifiable real-world events (see also Armson et al., 2016; St. Jacques, Montgomery, \& Schacter, 2015). We found that memory for the fine-grained spatiotemporal structure of a realworld event persists over months in both younger and older adults, and that increased lure susceptibility in older adults reverses the classic pattern of item and associative 
memory changes observed in laboratory studies of aging. Future work in this vein will contribute to understanding normal versus pathological memory changes in aging and how they manifest in day-to-day life. 


\section{References}

Adamo, D. E., Briceno, E. M., Sindone, J. A., Alexander, N. B., \& Moffat, S. D. (2012). Age differences in virtual environment and real world path integration. Frontiers in Aging Neuroscience, 4(SEP), 1-9. http://doi.org/10.3389/fnagi.2012.00026

Aggleton, J. P., \& Waskett, L. (1999). The ability of odours to serve as state-dependent cues for real-world memories: Can Viking smells aid the recall of Viking experiences? British Journal of Psychology, 90, 1-7. http://doi.org/10.1348/000712699161170

Aghajan, Z. M., Acharya, L., Moore, J. J., Cushman, J. D., Vuong, C., \& Mehta, M. R. (2015). Impaired spatial selectivity and intact phase precession in two-dimensional virtual reality. Nature Neuroscience, 18(1), 121-128. http://doi.org/10.1038/nn.3884

Allen, G. L., Kirasic, K. C., Rashotte, M. A., \& Haun, D. B. M. (2004). Aging and path integration skill: Kinesthetic and vestibular contributions to wayfinding. Perception and Psychophysics, 66(1), 170-179. http://doi.org/10.3758/BF03194870

Allen, T.A, Morris, AM., Stark, S.M, Fortin, N.J, \& Stark, C.E.L. (2015). Memory for sequences of events impaired in typical aging. Learn Mem, 22(3), 138-148. http://doi.org/10.1101//m.036301.114

Armson, M. J., Abdi, H., \& Levine, B. (2016). Bridging naturalistic and laboratory assessment of memory: the Baycrest mask fit test. Memory, $O(0), 1-10$. http://doi.org/10.1080/09658211.2016.1241281 
Bates, D., Mächler, M., Bolker, B., \& Walker, S. (2015). Fitting Linear Mixed-Effects Models using Ime4. Journal of Statistical Software, 67(1). http://doi.org/10.18637/jss.v067.i01

Bender, A. R., Naveh-Benjamin, M., \& Raz, N. (2010). Associative Deficit in Recognition Memory in a Lifespan Sample of Healthy Adults. Psychology and Aging, 25(4), 940-948. http://doi.org/10.1037/a0020595

Bontempi, B., Laurent-Demir, C., Destrade, C., \& Jaffard, R. (1999). Time-dependent reorganization of brain circuitry underlying long-term memory storage. Nature, 400(August), 671-675. http://doi.org/10.1038/23270

Brunec, I. K., Ozubko, J. D., Barense, M. D., \& Moscovitch, M. (2017). Recollectiondependent memory for event duration in large-scale spatial navigation. Learning \& Memory, 24, 104-114.

Burke, S. N., Wallace, J. L., Hartzell, A. L., Nematollahi, S., Plange, K., \& Barnes, C. A. (2011). Age-associated deficits in pattern separation functions of the perirhinal cortex: A cross-species consensus. Behavioral Neuroscience, 125(6), 836-47. http://doi.org/10.1037/a0026238

Buzsáki, G., \& Llinás, R. (2017). Space and Time in the brain. Science, 358, 482-485.

Campbell, K. L., Trelle, A., \& Hasher, L. (2014). Hyper-binding across time: Age differences in the effect of temporal proximity on paired-associate learning. Journal 
of Experimental Psychology: Learning Memory and Cognition, 40(1), 300-305. http://doi.org/10.1037/a0034109

Castel, A.D. (2005). Memory for grocery prices in younger and older adults: The role of schematic support. Psychology and Aging, 20(4), 718-721.

Cheke, L. G. (2016). What - where - when memory and encoding strategies in healthy aging. Learning \& Memory, 121-126.

Chen, H.-Y., Gilmore, A. W., Nelson, S. M., \& McDermott, K. B. (2017). Are there Multiple Kinds of Episodic Memory? An fMRI Investigation Comparing Autobiographical and Recognition Memory Tasks. The Journal of Neuroscience, 37(10), 1534-16. http://doi.org/10.1523/JNEUROSCI.1534-16.2017

Cohen, J. (1992). A power primer. Psychological Bulletin, 112(1), 155-159. http://doi.org/10.1037/0033-2909.112.1.155

Cohn, M., Emrich, S. M., \& Moscovitch, M. (2008). Age-related deficits in associative memory: the influence of impaired strategic retrieval. Psychology and Aging, 23(1), 93-103. http://doi.org/10.1037/0882-7974.23.1.93

Cornwell, B. R., Johnson, L. L., Holroyd, T., Carver, F. W., \& Grillon, C. (2008). Human Hippocampal and Parahippocampal Theta during Goal-Directed Spatial Navigation Predicts Performance on a Virtual Morris Water Maze. Journal of Neuroscience, 28(23), 5983-5990. http://doi.org/10.1523/JNEUROSCI.5001-07.2008 
Craik, F. (1986). A functional account of age differences in memory. Human Memory and Cognitive Capabilities: Mechanisms and Performances, (April), 409-22.

Craik, F. I. M., \& Schloerscheidt, A. M. (2011). Age-related differences in recognition memory: Effects of materials and context change. Psychology and Aging, 26(3), 671-677. http://doi.org/10.1037/a0022203

Curran, T., \& Friedman, W. J. (2003). Differentiating location- and distance-based processes in memory for time: An ERP study. Psychonomic Bulletin \& Review, 10(3), 711-717. http://doi.org/10.3758/BF03196536

Daum, I., Graber, S., Schugens, M. ., \& Mayes, A. . (1996). Memory dysfunction of the frontal type in normal ageing. NeuroReport. Retrieved from http://ovidsp.ovid.com/ovidweb.cgi?T=JS\&PAGE=reference\&D=emed4\&NEWS=N $\& A N=1996367879$

Dulas, M. R., Newsome, R. N., \& Duarte, A. (2011). The effects of aging on ERP correlates of source memory retrieval for self-referential information. Brain Research, 1377, 84-100. http://doi.org/10.1016/j.brainres.2010.12.087

Dumas, J. A., \& Hartman, M. (2003). Adult age differences in temporal and item memory. Psychology and Aging, 18(3), 573-586. http://doi.org/10.1037/08827974.18.3.573 
Ekstrom, A. D., Copara, M. S., Isham, E. a, Wang, W., \& Yonelinas, A. P. (2011).

Dissociable networks involved in spatial and temporal order source retrieval. Neurolmage, 56(3), 1803-13. http://doi.org/10.1016/j.neuroimage.2011.02.033

Eichenbaum, H., \& Cohen, N. J. (2014). Can We Reconcile the Declarative Memory and Spatial Navigation Views on Hippocampal Function? Neuron, 83(4), 764-770. http://doi.org/10.1016/j.neuron.2014.07.032

Etchamendy, N., Konishi, K., Pike, G. B., Marighetto, A., \& Bohbot, V. D. (2012). Evidence for a virtual human analog of a rodent relational memory task: A study of aging and fMRI in young adults. Hippocampus, 22(4), 869-880. http://doi.org/10.1002/hipo.20948

Fabiani, M., \& Friedman, D. (1997). Dissociations between memory for temporal order and recognition memory in aging. Neuropsychologia, 35(2).

Griffiths, B., Mazaheri, A., Debener, S., \& Hanslmayr, S. (2016). Brain oscillations track the formation of episodic memories in the real world. Neuroimage, 143, 256-266. http://doi.org/10.1101/042929

Gruber, M. J., Gelman, B. D., \& Ranganath, C. (2014). States of Curiosity Modulate Hippocampus-Dependent Learning via the Dopaminergic Circuit. Neuron, 84(2), 486-496. http://doi.org/10.1016/j.neuron.2014.08.060

Hashtroudi, S., Johnson, M. K., \& Chrosniak. (1989). Aging and Source Monitoring. Psychology and Aging, 4(1), 106-112. 
Hashtroudi, S., Parker, E. S., Luis, J. D., \& Reisen, C. A. (1989). Generation and elaboration in older adults. Experimental Aging Research, 15(1-2), 73-78. http://doi.org/10.1080/03610738908259760

Hassabis, D., Kumaran, D., Vann, S. D., \& Maguire, E. a. (2007). Patients with hippocampal amnesia cannot imagine new experiences. Proceedings of the National Academy of Sciences of the United States of America, 104(5), 1726-31. http://doi.org/10.1073/pnas.0610561104

Hegarty, M., Montello, D. R., Richardson, A. E., Ishikawa, T., \& Lovelace, K. (2006). Spatial abilities at different scales: Individual differences in aptitude-test performance and spatial-layout learning. Intelligence, 34(2), 151-176. http://doi.org/10.1016/j.intell.2005.09.005

Henkel, L. a. (2014). Point-and-Shoot Memories: The Influence of Taking Photos on Memory for a Museum Tour. Psychological Science, (December). http://doi.org/10.1177/0956797613504438

Hess, T. M., Germain, C. M., Swaim, E. L., \& Osowski, N. L. (2009). Aging and selective engagement: The moderating impact of motivation on older adults' resource utilization. Journals of Gerontology - Series B Psychological Sciences and Social Sciences, 64(4), 447-456. http://doi.org/10.1093/geronb/gbp020 
Howard, M. W., \& Kahana, M. J. (2002). A Distributed Representation of Temporal Context. Journal of Mathematical Psychology, 46(3), 269-299. http://doi.org/10.1006/jmps.2001.1388

Jacoby, L. L. (1991). A process dissociation framework: Separating Automatic from intentional uses of memory. Journal of Memory and Language, 30, 513-541.

Johnson, S. A., Turner, S. M., Santacroce, L. A., Carty, K. N., Shafiq, L., Bizon, J. L., ... Burke, S. N. (2017). Rodent age-related impairments in discriminating perceptually similar objects parallel those observed in humans. Hippocampus, 27(7), 759-776. http://doi.org/10.1002/hipo.22729

Kaplan, R., Doeller, C. F., Barnes, G. R., Litvak, V., Düzel, E., Bandettini, P. a, \& Burgess, N. (2012). Movement-related theta rhythm in humans: coordinating selfdirected hippocampal learning. PLoS Biology, 10(2), e1001267. http://doi.org/10.1371/journal.pbio.1001267

Kausler, D. H., \& Wiley, J. G. (1990). Temporal Memory and Content Memory for Actions: Adults age differences in acquisition and retention. Experimental Aging Research, 16(3), 147-150. http://doi.org/10.1080/07340669008251542

Koutstaal, W., \& Schacter, D. L. (1997). Gist-Based False Recognition of Pictures in Older and Younger Adults. Journal of Memory and Language, 37(4), 555-583. http://doi.org/10.1006/jmla.1997.2529 
Kroenke, K., Spitzer, R. L., \& Williams, J. B. W. (2001). The PHQ-9. Journal of General Internal Medicine, 16(9), 606-613. http://doi.org/10.1046/j.15251497.2001.016009606.x

Kuznetsova, A., Brockhoff, P., \& Christensen, R. (2016). ImerTest: Tests in Linear Mixed Effects Models. R Package Version, 3.0.0, https://cran.rproject.org/package=ImerTest. Retrieved from https://cran.rproject.org/package=ImerTest

Levine, B., Svoboda, E., Hay, J. F., Winocur, G., \& Moscovitch, M. (2002). Aging and autobiographical memory: Dissociating episodic from semantic retrieval. Psychology and Aging, 17(4), 677-689. http://doi.org/10.1037//0882-7974.17.4.677

Luke, S. G. (2017). Evaluating significance in linear mixed-effects models in R. Behavior Research Methods, 49(4), 1494-1502. http://doi.org/10.3758/s13428-016-0809-y

Macmillan, N. A., \& Creelman, C. D. (2005). Detection theory: A user's guide (2nd ed.). Mahwah, NJ: Erlbaum.

Mcgillivray, S., Murayama, K., \& Castel, A. D. (2015). Thirst for Knowledge : The Effects of Curiosity and Interest on Memory in Younger and Older Adults. Psychology and Aging, 30(4), 1-8. http://doi.org/10.1037/a0039801

Naveh-Benjamin, M. (1990). Coding of temporal order information: An automatic process? Journal of Experimental Psychology: Learning, Memory, and Cognition, 16(1), 117-126. http://doi.org/10.1037/0278-7393.16.1.117 
Naveh-Benjamin, M. (2000). Adult Age Differences in Memory Performance: Tests of an Associative Deficit Hypothesis. Journal of Experimental Psychology: Learning, Memory, and Cognilion, 26(5), 1170-1187. http://doi.org/10.1037//02787393.26.5.1170

Naveh-Benjamin, M., \& Craik, F. I. M. (1995). Memory for context and its use in item memory: Comparisons of younger and older persons. Psychology and Aging, 10(2), 284-293.

Newman, M. C., Allen, J. J., \& Kaszniak, A. W. (2001). Tasks for assessing memory for temporal order versus memory for items in aging. Aging, Neuropsychology, and Cognition, 8(1), 72-78. http://doi.org/10.1076/anec.8.1.72.849

Nielson, D. M., Smith, T. A., Sreekumar, V., Dennis, S., \& Sederberg, P. B. (2015). Human hippocampus represents space and time during retrieval of real-world memories. Proceedings of the National Academy of Sciences of the United States of America, 112(35), 11078-83. http://doi.org/10.1073/pnas.1507104112

Old, S. R., \& Naveh-Benjamin, M. (2008). Differential effects of age on item and associative measures of memory: a meta-analysis. Psychology and Aging, 23(1), 104-118. http://doi.org/10.1037/0882-7974.23.1.104

Perfect, T. J., \& Dasgupta, Z. R. R. (1997). What underlies the deficit in reported recollective experience in old age? Memory \& Cognition, 25(6), 849-858. http://doi.org/10.3758/BF03211329 
Perlmutter, M., Metzger, R., Nezworski, T., \& Miller, K. (1981). Spatial and temporal memory in 20 to 60 year olds. Journal of Gerontology, 36(1), 59-65. http://doi.org/10.1093/geronj/36.1.59

Pidgeon, L. M., \& Morcom, A. M. (2014). Age-related increases in false recognition: The role of perceptual and conceptual similarity. Frontiers in Aging Neuroscience, 6(OCT), 1-17. http://doi.org/10.3389/fnagi.2014.00283

Plancher, G., Gyselinck, V., Nicolas, S., \& Piolino, P. (2010). Age effect on components of episodic memory and feature binding: A virtual reality study. Neuropsychology, 24(3), 379-90. http://doi.org/10.1037/a0018680.

Plancher, G., Tirard, A., Gyselinck, V., Nicolas, S., \& Piolino, P. (2012). Using virtual reality to characterize episodic memory profiles in amnestic mild cognitive impairment and Alzheimer's disease: Influence of active and passive encoding. Neuropsychologia, 50(5), 592-602. http://doi.org/10.1016/j.neuropsychologia.2011.12.013

Ranganath, C., \& Hsieh, L.-T. (2016). The hippocampus: a special place for time. Annals of the New York Academy of Sciences, 1-18. http://doi.org/10.1111/nyas. 13043

Raz, N., Lindenberger, U., Rodrigue, K. M., Kennedy, K. M., Head, D., Williamson, A., ... Acker, J. D. (2005). Regional brain changes in aging healthy adults: general 
trends, individual differences and modifiers. Cerebral Cortex (New York, N.Y.: 1991), 15(11), 1676-89. http://doi.org/10.1093/cercor/bhi044

Reagh, Z. M., Ho, H. D., Leal, S. L., Noche, J. a, Chun, A., Murray, E. a, \& Yassa, M. a. (2016). Greater loss of object than spatial mnemonic discrimination in aged adults. Hippocampus, 422, 417-422. http://doi.org/10.1002/hipo.22562

Roberts, J. M., Ly, M., Murray, E., \& Yassa, M. A. (2014). Temporal discrimination deficits as a function of lag interference in older adults. Hippocampus, 24(10), 1189-1196. http://doi.org/10.1002/hipo.22303

Robin, J., \& Moscovitch, M. (2017). Familiar Real-World Spatial Cues Provide Memory Benefits in Older and Younger Adults. Psychology and Aging. http://doi.org/10.1037/pag0000162

Robin, J., Wynn, J., \& Moscovitch, M. (2015). The spatial scaffold: The effects of spatial context on memory for events. Journal of Experimental Psychology: Learning, Memory, and Cognition, 42(2), 308-315. http://doi.org/http://dx.doi.org/10.1037/xIm0000167

Ryan, L., Cardoza, J. A., Barense, M. D., Kawa, K. H., Wallentin-Flores, J., Arnold, W. T., \& Alexander, G. E. (2012). Age-related impairment in a complex object discrimination task that engages perirhinal cortex. Hippocampus, 22(10), 19781989. http://doi.org/10.1002/hipo.22069 
Schacter, D. L., Koutstaal, W., Johnson, M. K., Gross, M. S., \& Angell, K. E. (1997). False recollection induced by photographs: a comparison of older and younger adults. Psychology and Aging, 12(2), 203-215. http://doi.org/10.1037/08827974.12.2.203

Schmitter-Edgecombe, M., \& Simpson, A. L. (2001). Effects of Age and Intentionality on Content Memory and Temporal Memory for Performed Activities. Aging, Neuropsychology, and Cognition, 8(2), 81-97. http://doi.org/10.1076/anec.8.2.81.844

Shapiro, M. L., Kennedy, P. J., \& Ferbinteanu, J. (2006). Representing episodes in the mammalian brain. Current Opinion in Neurobiology, 16(6), 701-709. http://doi.org/10.1016/j.conb.2006.08.017

Sharps, M. J., \& Gollin, E. S. (1987). Memory for object locations in younger and elderly adults. Journal of Gerontology, 42(3), 336-341.

Sheldon, S., \& Levine, B. (2013). Same as it ever was: vividness modulates the similarities and differences between the neural networks that support retrieving remote and recent autobiographical memories. Neurolmage, 83, 880-91. http://doi.org/10.1016/j.neuroimage.2013.06.082

Skinner, E. I., \& Fernandes, M. A. (2009). Age-related changes in the use of study context to increase recollection. Neuropsychology, Development, and Cognition. 
Section B, Aging, Neuropsychology and Cognition, 16(4), 377-400.

http://doi.org/10.1080/13825580802573052

Skowronski, J. J., Walker, W. R., \& Betz, A. L. (2003). Ordering our world : An examination of time in autobiographical memory. Memory, 11(3), 247-261. http://doi.org/10.1080/09658210244000009

Spencer, W. D., \& Raz, N. (1995). Differential effects of aging on memory for content and context: A meta-analysis. Psychology and Aging.

St Jacques, P. L., Montgomery, D., \& Schacter, D. L. (2015). Modifying memory for a museum tour in older adults: Reactivation-related updating that enhances and distorts memory is reduced in ageing. Memory, 23(July 2014), 1-12. http://doi.org/10.1080/09658211.2014.933241

St. Jacques, P. L., Olm, C., \& Schacter, D. L. (2013). Neural mechanisms of reactivation-induced updating that enhance and distort memory. Proceedings of the National Academy of Sciences, 110(49). http://doi.org/10.1073/pnas.1319630110

St Jacques, P., Rubin, D. C., LaBar, K. S., \& Cabeza, R. (2008). The short and long of it: neural correlates of temporal-order memory for autobiographical events. Journal of Cognitive Neuroscience, 20(7), 1327-41. http://doi.org/10.1162/jocn.2008.20091

St. Jacques, P. L., \& Schacter, D. L. (2013). Modifying memory: selectively enhancing and updating personal memories for a museum tour by reactivating them. Psychological Science, 24(4), 537-43. http://doi.org/10.1177/0956797612457377 
St-Laurent, M., Moscovitch, M., \& McAndrews, M. P. (2016). The retrieval of perceptual memory details depends on right hippocampal integrity and activation. Cortex, 84 , 15-33. http://doi.org/10.1016/j.cortex.2016.08.010

Stark, S. M., Reagh, Z. M., Yassa, M. A., \& Stark, C. E. L. (2017). What's in a context? Cautions, limitations, and potential paths forward. Neuroscience Letters, 1-11. http://doi.org/10.1016/j.neulet.2017.05.022

Steiger, J. H. (1980). Tests for comparing elements of a correlation matrix. Psychological Bulletin, 87, 245-251.

Taube, J. S., Valerio, S., \& Yoder, R. . (2013). Is Navigation in Virtual Reality with fMRI Really Navigation? Journal of Cognitive Neuroscience, 25(7), 1008-1019. http://doi.org/10.1162/jocn

Thome, A., Marrone, D. F., Ellmore, T. M., Chawla, M. K., Lipa, P., Ramirez-Amaya, V., ... Barnes, C. A. (2017). Evidence for an Evolutionarily Conserved Memory Coding Scheme in the Mammalian Hippocampus. The Journal of Neuroscience, 37(10), 3057-16. http://doi.org/10.1523/JNEUROSCI.3057-16.2017

Tolentino, J. C., Pirogovsky, E., Luu, T., Toner, C. K., \& Gilbert, P. E. (2012). The effect of interference on temporal order memory for random and fixed sequences in nondemented older adults. Learning \& Memory, 19(6), 251-255. http://doi.org/10.1101//m.026062.112 
Troyer, A. K., Murphy, K. J., Anderson, N. D., Craik, F. I., Moscovitch, M., Maione, A., \& Gao, F. (2012). Associative recognition in mild cognitive impairment: relationship to hippocampal volume and apolipoprotein E. Neuropsychologia, 50(14), 3721-3728. http://doi.org/10.1016/j.neuropsychologia.2012.10.018

Tulving, E. (1972). Episodic and semantic memory. In E. Tulving \& W. Donaldson (Eds.), Organization of memory (pp. 381-402). Oxford, England. Academic Press.

Tzeng, O. J., \& Cotton, B. (1980). A study-phase retrieval model of temporal coding. Journal of Experimental Psychology: Human Learning \& Memory, 6(6), 705-716. http://doi.org/10.1037/0278-7393.6.6.705

Underwood, B. J. (1977). Temporal codes for memories: Issues and problems. Hillsdale, NJ: Erlbaum.

Uttl, B., \& Graf, P. (1993). Episodic Spatial Memory in Adulthood. Psychology and Aging, 8(2), 257-273. http://doi.org/10.1037/0882-7974.8.2.257

Vilberg, K. L., \& Rugg, M. D. (2009). Functional significance of retrieval-related activity in lateral parietal cortex: evidence from fMRI and ERPs. Human Brain Mapping, 30(5), 1490-1501. http://doi.org/10.1002/hbm.20618

Voss, J. L., Gonsalves, B. D., Federmeier, K. D., Tranel, D., \& Cohen, N. J. (2011). Hippocampal brain-network coordination during volitional exploratory behavior enhances learning. Nature Neuroscience, 14(1), 115-20. http://doi.org/10.1038/nn.2693 
Wang, R. F., \& Simons, D. J. (1999). Active and passive scene recognition across views. Cognition, 70(2), 191-210. http://doi.org/10.1016/S0010-0277(99)00012-8

Winocur, G., Moscovitch, M., Caruana, D. A., \& Binns, M. A. (2005). Retrograde amnesia in rats with lesions to the hippocampus on a test of spatial memory. Neuropsychologia, 43(11), 1580-1590. http://doi.org/10.1016/j.neuropsychologia.2005.01.013

Yeung, L.-K., Ryan, J. D., Cowell, R. A, \& Barense, M. D. (2013). Recognition memory impairments caused by false recognition of novel objects. Journal of Experimental Psychology. General, 142(4), 1384-97. http://doi.org/10.1037/a0034021

Zacks, R. T., Hasher, L., Alba, J. W., Sanft, H., \& Rose, K. C. (1984). Is temporal order encoded automatically? Memory \& Cognition, 12(4), 387-394.

Zisch, F. E., Gage, S., \& Spiers, H. J. (2014). Navigating the museum. In N. Levent \& A. Pascual-Leone (Eds.), The multi-sensory museum: Cross-disciplinary perspectives on touch, sound, smell, memory, and space. 